\title{
Deux ateliers du poème pour « essayer dire » en français langue étrangère
}

Two workshops of the poem to "try say" in French as a foreign language

\section{Olivier Mouginot}

\section{(2) OpenEdition}

\section{Journals}

Édition électronique

URL : http://journals.openedition.org/pratiques/5254

DOI : 10.4000/pratiques.5254

ISSN : 2425-2042

Éditeur

Centre de recherche sur les médiations (CREM)

Référence électronique

Olivier Mouginot, « Deux ateliers du poème pour « essayer dire » en français langue étrangère », Pratiques [En ligne], 179-180 | 2018, mis en ligne le 31 décembre 2018, consulté le 10 décembre 2020 URL : http://journals.openedition.org/pratiques/5254; DOI : https://doi.org/10.4000/pratiques.5254

Ce document a été généré automatiquement le 10 décembre 2020.

(c) Tous droits réservés 


\title{
Deux ateliers du poème pour « essayer dire » en français langue étrangère
}

Two workshops of the poem to "try say" in French as a foreign language

\author{
Olivier Mouginot
}

Le Cadre européen commun de référence pour les langues (CERCL, 2001) définit l'apprenant d'une langue étrangère comme un «acteur social». Dans une perspective méthodologique dite actionnelle, la compétence de l'apprenant à «communiquer langagièrement » dans différents «domaines » (éducationnel, professionnel, public, personnel) constitue l'objectif affiché des apprentissages linguistiques contemporains. Dans cette conception, l'individu semble primer sur le sujet, l'individuation sur la subjectivation, l'action sur l'écoute et le dire. Au passage, la littérature, seulement vue comme texte, valeur ou document, est laissée sans réelle(s) voix au chapitre. Ces restrictions pourraient expliquer en partie pourquoi la didactique du français langue étrangère (FLE) dépense aujourd'hui encore une énergie considérable à vouloir clarifier le (s) rôle(s) à jouer de la littérature en classe de langue. Malgré des synthèses qui marquent un pas décisif vers une transmission d'ordre expérientielle (par exemple, Pierra, 2006; Siouffi, 2007; Godard, Havard \& Rollinat-Levasseur, 2011; Godard, 2015), l'instrumentalisation de la littérature reste toutefois un paradigme méthodologique en vigueur jusque dans des "activités langagières" - pluriel emprunté au CECRL pour désigner la réception, la production, l'interaction et la médiation - qui devraient normalement constituer des échappatoires au communicativisme: écriture créative, chanson, slam, théâtre, etc. Ce qui manque peut-être actuellement le plus à la didactique du FLE, ce sont des continuités notionnelles/expérientielles fortes de type apprentissagesubjectivation, langues-langage, langage-corps, écriture-oralité, ou encore arts du langage-vie quotidienne. La segmentation de la parole (parlée, lue, écrite, évaluée) redouble celle du sujet (sujet plurilingue, sujet-apprenant, sujet-lecteur, sujet-écrivant) dont l'activité langagière fait l'objet d'un méthodique saucissonnage. 
2 Dans le même temps, des agencements très divers d'expériences individuelles et collectives avec les arts du langage - dénommés « ateliers du dire » dans notre recherche doctorale - offrent à voir d'étonnantes propositions associant littératures et langues de vie. Au contact de la diversité des plurilinguismes individuels, ces pratiques littératiées vivantes s'inventent aussi en français comme langue étrangère, à l'occasion de parcours d'apprentissage, de formation ou d'intégration. Modifiant les représentations et les habitus rattachés d'ordinaire à la littérature et à la "poésie » en classe de langue, ces ateliers du dire sont très souvent des ateliers de littératures et, plus particulièrement, des ateliers avec des poèmes - nous verrons plus loin comment ils sont aussi et surtout des ateliers $d u$ poème. Cette fréquence ne doit toutefois rien au hasard: comme nombre de leurs cousins maternels, si les ateliers en langue(s) étrangère(s) s'appuient sur des œuvres littéraires, c'est qu'ils y puisent un faire langage exemplaire - en référence à cette invitation de M. de Certeau (1990, p. 204) d'« un langage à faire et non plus seulement à entendre ». De nature tactique, ce faire langage des ateliers du dire peut surtout compter sur un «faire œuvre avec les œuvres» (Martin, 2005; 2016), c'est-à-dire sur une dynamique réénonciative et transsubjective. Avant d'illustrer notre propos à travers deux expériences d'atelier, il nous faut préciser ici quelle exemplarité des œuvres de langage est privilégiée.

\section{Ateliers du dire, ateliers du poème}

Un atelier du dire, ce peut être toute expérience qui invite à « essayer dire » en langue(s) étrangère(s). Nous empruntons cette formule à $\mathrm{S}$. Beckett, déclinée dans les pages de Cap au pire (1991). Ces deux infinitifs se voient associés pour définir l'activité langage de l'être humain, le processus même de sa subjectivation «dans et par le langage » (Benveniste, 1966). « Essayer dire » en langue(s) étrangère(s) ne change aucunement la donne à partir du moment où il s'agit de profiter de l'élan énonciatif et vocal d'œuvres de langage spécifiques, c'est-à-dire dans lesquelles "un sujet s'inscrit au maximum dans son discours, inscrit au maximum sa situation dans un discours» (Meschonnic, 2009, p. 73). L'exemplarité des corpus littéraires d'ateliers du dire tient peut-être à cette recherche de lieux dans le langage " où s'invente et se défend le sujet » (Meschonnic, 2008, p. 121). En didactique du français, Ph. Païni $(2005$, p. 46) fait du poème « ce qu'il y a de plus langage dans le langage, un maximum, pas un écart » - ajoutant qu' « il y a dans le poème cette évidence que ni le contenu ni la forme, ni leur séparation ne suffisent ni pour le faire ni pour le lire ». Tout atelier du dire entendu comme atelier du poème peut d'ailleurs être vu comme expérience du bref dans la conception de G. Dessons (2015, p. 143) - «le moment où le langage passe en mode poème » pour chercher la « voix juste ». En atelier, la transsubjectivité littéraire ou poétique montre en quelque sorte la voie en servant plus largement le projet langagier d'un « essayer dire » qui vise des passages de voix. Dans cette perspective, il s'agit alors de distinguer en pratique atelier du poème et atelier de poésie: «le poème, et le bref, ne se trouvent ni toujours, ni obligatoirement, dans le genre "poétique". Il y a du poème dans le roman, dans la nouvelle, dans le théâtre, dans la prose scientifique aussi, là où du spécifique émerge dans le langage. » (ibid., p. 131).

Il faut déplorer que les ateliers du dire comme pratiques langagières spécifiques intéressent peu la recherche en didactique des langues et du plurilinguisme (Pierra, 2006; Vorger, 2011; Mathis, 2013; Aden, 2013; Dos Santos, 2013). Amorcée en 2014, notre recherche doctorale fait l'hypothèse de nombreux enseignements à tirer de ces pratiques 
en langue(s) étrangère(s) dans le double but de remédier à leur marginalisation tout en contribuant à renforcer la didactique du FLE comme didactique relationnelle entre sujets d'une création culturelle et langagière toujours située et plurielle. Par leurs manières inédites d'imaginer de multiples "nœuds énonciatifs " à partir de la "puissance des œuvres " (Joubert, 2015), les ateliers du dire questionnent le parcours relationnel des enseignants comme des apprenants avec les œuvres littéraires. Par les déplacements théoriques et pratiques qu'ils impliquent - dynamique réénonciative en lieu et place d'une logique de substitution, revalorisation de l'oralité comme mode de signifier, ils incitent à des sorties méthodologiques privilégiant les essais d'écoute et de reprise de voix. G. Dessons $(2015$, p. 133) rappelle que « le problème pour un poème n'est pas [...] de "vouloir dire", mais d'agir dans une culture dont il procède et à l'invention de laquelle il contribue». Au-delà des gestes scolaires (expliquer, interpréter, participer) qui sont toujours des réponses attendues au poème, il va plutôt s'agir en atelier de faire comme le « sujet du poème » (Meschonnic, 2008, p. 188) - et non pas à la manière de tel poète ou de tel poème.

5 Nous proposons de rendre compte de ce "mode d'être dans le langage » (Dessons, 2015, p. 139) favorisé par les ateliers du dire à travers deux expériences en FLE. Premièrement, nous ferons référence à l'«atelier de l'illisible» de M. Depeursinge, conduit entre 2014-2015 dans une classe d'accueil de l'École de la Transition à Lausanne (Suisse). Enseignant de français, M. Depeursinge est par ailleurs l'auteur d'une thèse doctorale (2018) sur la lecture de textes poétiques contemporains avec des élèves en situation d'exclusion scolaire et des adolescents primo-arrivants. Dans un second temps, nous reviendrons sur un atelier au long cours (2004-2016), les «lectures de bouches» de P. Fontana, comédien et artiste plasticien français. Nous nous arrêterons en particulier sur l'expérience qu'il a conduite en partenariat avec l'association Emmaüs Solidarité (Paris) pendant plusieurs années.

\section{L'« atelier de l'illisible » de M. Depeursinge : « penser poème » pour écouter-dire}

6 «Peut-être qu'un poème commence quand on ne sait plus ce que signifie l'opposition entre facile et difficile.» (Meschonnic, 2006, p. 104) Telle semble la piste de travail empruntée par M. Depeursinge pour concevoir et conduire son « atelier de l'illisible». L'opposition disparait rapidement avec le détournement de la question professorale «qu'est-ce que ça veut dire? ». Au lieu d'inviter à détrousser le sens du poème - ici le poème de C. Prigent (2012), " (du neuf sur la schize) », extrait du recueil La vie moderne, M. Depeursinge remet à la fois en question et en jeu l'approche traditionnelle de la signification. Nous redonnons ici les quatre premiers vers du poème :

No touche (navigation virtuelle) ordi

Et vitrine tactique Ubicity qui

Te connaît sur le bout de tes doigts : plus

Transparent que le poisson électrocu

7 Par le choix du poème, M. Depeursinge prend au passage le double contrepied d'une " poésie » pour la classe de langue et d'une littérature pour les ateliers : «On dit textes d'expert, résistants, nécessitant un haut niveau de littératie, mais comme ces textes interrogent tout le monde sur notre rapport au sens, à l'interaction, c'est très fort avec tout le monde. » (Depeursinge \& Mouginot, 2017) Son attention se porte avec insistance 
sur l'expérience d'historicité propre à toute lecture. Ce qui le relie d'une certaine manière à A. Bernardet (2012, p. 10) quand ce dernier indique que « les phénomènes d'obscurité et d'illisibilité demeurent des critères mouvants et relatifs. Ils ressortissent moins à quelque essence supposée du littéraire [...] qu'à l'historicité des lectures elles-mêmes.» Cette historicité sert à la fois à répéter des savoir-faire et à en produire de nouveaux. C'est non seulement une méthodologie nouvelle qui s'invente à l'abord d'un tel poème, mais aussi une réflexivité différente qui fait des participants des lecteurs plus conscients et plus critiques des conditions de leur expérience d'apprentissage - et de leur apprentissage comme expérience. Il n'y a toutefois pas évacuation totale d'un traitement du sens dans cet « atelier de l'illisible ». La proposition de M. Depeursinge relèverait plutôt d'un certain bon sens attaché à un dosage expérientiel. En effet, son savoir-faire d'atelier consiste à substituer des expériences d'historicité à des cheminements scolaires. Ce qui n'est pas sans évoquer la dichotomie d'H. Meschonnic (2006, p. 134) entre "lecture-mime» et «lecture-historicité ». Cette seconde est faite d'une pluralité de questions qui ne sont pas connues à l'avance et qui ne sont pas des injonctions, des vérifications ou des attentes. Dans cette perspective, $M$. Depeursinge teste en atelier la validité de l'hypothèse suivante d'H. Meschonnic (ibid., p. 134-135) : "Il n'y a que les questions qui lisent. Non pour chercher des réponses, mais pour voir comment sont posées les questions. Dès qu'on lit sans question, on ne lit plus, on est, à l'inverse, dévoré par l'“objet" de la lecture. »

Pour lire véritablement l'« illisible ", c'est-à-dire tout à la fois choisir les bonnes questions à (se) poser, essayer d'écouter le poème et réfléchir à la portée de cette écoute, M. Depeursinge met en place un dispositif de prise de notes collective au moyen de la vidéo-projection, laquelle autorise une dictée en direct qui regroupe toutes les « retraductions, reformulations, récritures» (Depeursinge, 2018) des participants. Cette souplesse réflexive au plus près de chaque segment du poème vient revitaliser l'activité de transmission littéraire en l'émancipant des schémas explicatifs et interprétatifs. Ce travail d'écoute très élaboré nous parait d'ailleurs impliquer en pratique un «penser poème ». Nous réénonçons ici la formule de G. Noiret (2011, p. 9), lequel définit le lecteur d'un poème comme celui qui aurait de l'empathie pour le rythme, « et pas uniquement le signifiant ou le signifié ». À partir de cette hypothèse, il oppose poésie et poème : « penser poésie suppose une réalité supérieure et induit des déclarations d'amour ou de haine, penser poème permet une approche qui gagne en perception des phénomènes langagiers ce qu'elle perd en hauteur métaphysique. » Il va même jusqu'à proposer une définition à la fois mathématique et « instable » du poème mais qui nous semble assez bien ajustée à la proposition de M. Depeursinge comme à notre propre conceptualisation de l'essai de voix en atelier :

À mon sens, un poème est la cristallisation d'une sorte de produit de facteurs, au nombre indéterminé, à la définition instable, où les composants ne multiplient pas forcément d'une manière identique, mais un produit de facteurs quand même. Sans forcer, je peux risquer cette formule: poème = (langage) (histoire poétique) (imaginaire) (monde) (pensée) (projet). (ibid.)

9 C'est presque une poétique que G. Noiret (ibid., p. 10) propose à la fin de sa démonstration facétieuse: «En règle générale, les poèmes qui me parlent mobilisent de trois à cinq facteurs. Quand ils en utilisent deux, ils relèvent de la notation. Quand ils combinent les six, ils souffrent de saturation [...]. » Il ne s'agit pas d'une échelle de valeurs, mais d'un carrefour de pistes ouvertes vers de multiples expériences d'écoute et d'écriture critique - des lectures écrites dans l'«atelier de l'illisible». Il nous semble que ce sont ces «facteurs» que M. Depeursinge essaie de faire éprouver aux jeunes participants de son 
atelier. Il reste que ce qui prime, c'est l'activité d'écouter-dire et non plus celle de questionner le dit en imposant toutes les valeurs de référence. Dès la première séance d'atelier, l'animateur formule d'ailleurs une résistance collective au sens du poème faisant de l'« illisible» l'affaire de tous : «J'annonce que ce texte me résiste à moi aussi, radicalement. Le point de départ est d'annoncer honnêtement une communauté de situations. Il y a un côté performatif, mais ce n'est pas de la poudre aux yeux. Ça demande en pratique peu d'explications. » (Depeursinge \& Mouginot, 2017).

10 M. Depeursinge évoque ici non seulement les conditions d'une écoute mais aussi et surtout les conditions d'une recherche d'égalité énonciative, d'égalité dans l'expérience de reprise des voix. Cette déclaration inaugurale sert aussi à introduire la consigne de son atelier qui relève concrètement d'un parcours à étapes où la compréhension du poème fait elle-même et dans le même temps l'objet d'une déconstruction méthodique sur ce que peut vouloir dire illisible sinon difficile. Une double différenciation global/local et objectif/subjectif permet non seulement une approche du poème mais aussi et surtout son écoute avec des moyens détournés qui ne sont plus seulement ceux de l'analyse de texte :

Comment on se débrouille à partir du moment où on arrête de considérer le poème seulement comme un texte, comme une configuration générale? Dans le cheminement avec le texte, il y a des choses qui nous frappent, nous happent. Des choses tout à fait compréhensibles localement si nous contredisons la logique du texte. Ou bien alors il y a d'autres éléments qui nous parlent, d'un point de vue subjectif. (ibid.)

11 Au cœur de son activité critique, l'« atelier de l'illisible » produit une lecture chorale qui continue le poème sans cesser d'y retourner - une sorte de circuit court réénonciatif et transsubjectif (quand l'essai de voix transformé serait plutôt de l'ordre d'un circuit long). Une telle pratique est notamment permise par une "défamiliarisation ", une expérience d'étrangeté discursive suscitant un geste de réponse, presque un geste réflexe :

[Les élèves] produisent des modes de questionnement non seulement sur le texte mais aussi par-delà le texte, sur ce que le texte leur fait dire d'eux-mêmes. Le texte déplace leur propre point de vue. Parce qu'il déstabilise les représentations communes, nos manières de dire habituelles. Le texte envisage ou engage des choses tout à fait communes mais d'une manière stylistique différente qui « étrangéifie » tous nos modes rassurants, habituels. En retour, l'effet qu'il a, c'est de nous pousser à envisager, penser, ressentir autrement des choses assez communes. Je pars de ce point commun-là. Entre l'univers familier du lecteur et une défamiliarisation du texte. (ibid.)

Cette manière d'appropriation assez directe est d'ordre relationnel et c'est à ce titre qu'elle travaille le régime rythmique de l'œuvre sans rien en laisser paraitre. Nous voulons dire par là que cette proposition de lecture mime l'activité rythmique qui est un frottement de voix à l'intérieur d'un même continu discursif. Ici, l'écoute est un mode de passage de voix croisant l'inédit du poème et l'inédit du à-redire des participants :

Ce que j'ai observé dans les ateliers que j'ai menés, c'est qu'on récupère des choses qui seraient rejetées dans un cadre scolaire parce que non vérifiables mais qui jouent avec le poème et permettent de dire des choses assez formidables sur la relation entre le lecteur et le poème. Et, au-delà, sur le texte lui-même, en révélant des points de vue inédits sur ses dimensions, aspects ou fragments plus compréhensibles, qui sans cela, sans ce jeu, passeraient à côté du texte. (ibid.)

13 Comme le montre le relevé final des participants, la lecture peut alors devenir une création discursive à part entière - ainsi que le dit H. Meschonnic (2006, p. 135) : «[...] en 
tant qu'acte, et que pratique, [la lecture] a ses créations propres, de sens, et de sens du sens. » Prenons l'exemple d'un fragment extrait du dernier vers du poème de C. Prigent « Mage où est le monde en vrai nunc \& ibi? »:

Où est le monde en vrai?

Le monde en vrai, c'est dans la réalité. Il y a des gens qui vivent « au ciel », " la tête dans les nuages", pas les pieds sur terre. Le monde, c'est pas le rêve. Ou alors, ce n'est pas vivre « dans le futur ». Le monde en vrai, c'est dans le présent.

Le monde en vrai, c'est ici. Ici, dans la classe ? Non, c'est partout, c'est le monde.

Le faire atelier de M. Depeursinge modifie non seulement les usages scolaires liés à la compréhension et à l'interprétation mais aussi ceux ayant trait à l'exploitation du poème. L'« essayer dire » permis par cette écoute plurielle n'est pas ici un faire dire grâce auquel l'expérience $d u$ poème s'achèverait par la création d'un clone plus ou moins viable. L'atelier du dire sort de cette tension entre didactisme et ludisme pour repenser les invitations à faire corps avec les œuvres de langage. En l'espèce, M. Depeursinge cherche peut-être à éviter les difficultés qui se présentent parfois en matière d'oralisation ou d'écriture en classe de langue étrangère, notamment quand de telles continuations se voient accolées de manière automatique au travail sur un texte littéraire. La notation individuelle et collective proposée par M. Depeursinge, qui se traduit par des réécritures successives, ne conduit pas à une production quelconque qui viendrait avaliser la lecture du poème mais plutôt à une connaissance partagée :

ce texte va être repris à chaque séance. Je ne veux pas que ce soit juste un travail de transfert, d'équivalences, qui fait qu'on répond au poème par un autre poème. Travail qui fait qu'on reste dans un niveau d'indétermination. Le but, c'est aussi de travailler là où quelque chose peut se solidifier (ou pas) au sens du commun. (Depeursinge \& Mouginot, 2017)

\section{Les « lectures de bouches » de P. Fontana : «prendre corps » pour faire entendre sa voix}

15 P. Fontana définit ses «lectures de bouches » comme un atelier de lecture de textes littéraires et poétiques à voix hautes et enregistrées. Cet atelier s'adresse à des adultes qui sont parallèlement en situation d'apprentissage du FLE. Les « lectures de bouches » ont produit des traces singulières - dont le disque Luca Babel, Coup de cœur 2014 de l'Académie Charles Cros. Pour mieux comprendre cet atelier, peut-être faut-il repartir du propre étonnement de P. Fontana devant les possibilités offertes par la poésie de G. Luca :

Une des choses les plus intéressantes qui se passe dans cet atelier, est d'installer la poésie comme un exercice évident pour apprendre la langue française!

Ghérasim Luca est un pilier, sa poésie amène une certaine liberté car il permet en permanence de se détacher du sens, ce qui donne aux stagiaires, une sorte de désinhibition face à l'apprentissage de la langue. Cela les rend plus téméraires à expérimenter les sonorités délirantes de Luca. (Fontana, 2009-2011, 29/04/2010)

Il faut s'arrêter sur cette «évidence » et surtout sur la présence de poèmes de G. Luca dans un atelier à destination d'adultes nouvellement arrivés en France. G. Luca permet à P. Fontana et aux participants de l'atelier de "prendre corps » dans un discours - nous empruntons ce "prendre corps » au poème La fin du monde de G. Luca (2001, p. 287). En effet, cet atelier vient interroger ce que l'enseignement ne voit peut-être plus : du corps est requis dès qu'il y a discours à l'essai. Soit du corps et pas seulement du coffre (s'agissant de l'enseignant); de la voix dans le corps, du corps dans la voix, et pas 
seulement du cœur à l'ouvrage (s'agissant des apprenants ou des participants). S'appuyer sur de tels poèmes n'est pas un choix téméraire de la part de $\mathrm{P}$. Fontana, c'est continuer fidèlement la vie de l'œuvre de G. Luca. Car il faut avoir à l'esprit que la lecture à voix haute est une pratique spécifique chez le poète « apatride ». A. Velter (2001, p. I) rappelle que, dès son adolescence, G. Luca a « l'intuition que son pays, c'est son corps; que son identité, c'est sa voix». Une idée précise du niveau d'intensité vocale nécessaire est communiquée par le poète lui-même qui a donné de nombreux « récitals » de ses œuvres. S'inspirant directement de cette expérience langagière du poète, $P$. Fontana fait lire ses participants selon des modalités bien précises: un lire qui est un «façonner", un "modeler", un "sculpter ». Évoquant des travaux manuels ou physiques, ces verbes concrets ne renvoient pas seulement à des jeux d'articulation, des procédés de correction phonétique ou des entrainements prosodiques. Peut-être cette référence au verbe " éructer " - également emprunté à G. Luca - sort-elle totalement les «lectures de bouches » d'un enseignement linguistique :

Il y a une histoire de placer la bouche. Le son comme une sculpture. C'est ce que j'ai pris à Ghérasim Luca qui disait qu'il faut éructer les mots, les cracher, les former dans la bouche. J'ai cette image à faire passer aux participants. Cette idée de construire avec la bouche un autre langage. (Fontana \& Mouginot, 2017)

Éructation des mots, déploiement des phrases, apparition des rythmes: c'est une expérience de toute l'activité signifiante du langage qui s'échafaude. Tout l'intérêt des pratiques en langue étrangère développées par $P$. Fontana est en quelque sorte concentré dans cette autre formule: " une affaire de langues et de bouches » (Luca Babel, 2013). S'y dit en creux toute une histoire de réconciliation empirique dans l'espace de l'atelier: entre langues en présence, entre poèmes et langue française de tous les jours, entre langage et corps, etc. Ici la langue est d'abord l'organe de la parole - avant de renvoyer, au pluriel, au plurilinguisme spécifique de l'atelier. Dans ces «lectures de bouches », c'est donc un travail physique de la langue qui occupe les participants. Attentif aux autres, chacun travaille avec sa bouche. L'« essayer dire » est ce qui transforme les bouches avant même les discours. Comme si le dire était dans la bouche : « Le poème [...] devient de plus en plus présent dans les bouches des participants à l'atelier, les bouches commencent à se transformer. » (Fontana, 2009-2011, 27/01/2010) Mais l'atelier du dire n'est pas seulement une affaire de bouches. Tout le corps est embauché dans le faire langage de l'atelier. Cet autre compte rendu de P. Fontana montre que l'atelier du dire, ce peut être toujours plus d'expériences du corps-langage - ici avec le poème L'étranger de C. Baudelaire (2003) :

\footnotetext{
Nous avons commencé par un cours... de tai chi (!) donné par une des stagiaires qui le pratique tous les jours aux Buttes Chaumont! Ce temps inattendu était incroyable car l'ensemble des personnes présentes se sont mises à suivre les mouvements avec une disponibilité déconcertante.

J'en ai profité pour rebondir. La répétition d'une phrase en tai chi et sa précision m'a aidé à faire comprendre pourquoi je tenais tout le temps à rendre de plus en plus précis les sonorités des mots et en particulier dans les poèmes de Baudelaire. Ensuite, tout le monde a lu le poème L'étranger en même temps dans sa langue maternelle en faisant attention aux autres.

(P. Fontana, 2009-2011, 29/04/2010)
}

Dans les « lectures de bouches », l'écoute est au cœur de l'activité du dire. Elle n'est pas plus le commencement du dire que l'achèvement. Elle est une constante, un pôle magnétique de l'expérience langagière. Même les travaux phonétiques les plus rudimentaires proposés par P Fontana sont marqués par l'indissociation écouter-dire - à ne pas confondre avec le couple lire-écouter: l'intérêt porte à la fois sur comment 
produire les sons, comment les percevoir chez les autres. En ce sens, les «lectures de bouches" ne sont pas qu'un atelier de lecture à voix haute. Elles sont tout autant atelier d'écoute. Signalons que P. Fontana recourt à de nombreuses modalités d'écoute qui engagent des procédés d'enregistrement, faisant de l'écoute un carrefour expérientiel par lequel chaque essai de voix peut passer et repasser :

J'enregistre [les participants] tout le temps et il y a un travail permanent de réécoute. C'est très important. J'ai du matériel qui me permet d'enregistrer et d'écouter dans un même temps. Ils écoutent tout ce qu'ils font, tout ce qu'on fait ensemble. (Fontana \& Mouginot, 2017)

L'écoute devient alors ce démultiplicateur d'expériences et de savoirs. Puisque toute «lecture de bouche» est systématiquement un "arpentage» (Fontana, 2009-2011, 04/11/2010) à voix haute, l'enregistrement permet d'écouter et de dire indéfiniment à toutes les vitesses possibles. Il met l'écoute au cœur du dire et le dire au cœur de l'écoute :

On prend un poème et on commence à lire. Mais le lire c'est déjà organiser la lecture. Chacune une ligne, par exemple. Rapidement, je commence à organiser les voix. Les réponses. Les vitesses. Je dispose les gens. J'interviens dès le début. Il faut saisir les gens tout de suite. Les saisir au sens de les intéresser. Le plus important est de montrer tout de suite aux participants là où je veux aller. Ne pas cacher l'objectif. «On va tout de suite lire le texte. » « On va lire tous ensemble. » « On va tout de suite s'enregistrer. » « On va tout de suite s'écouter. » (Fontana \& Mouginot, 2017)

Concrètement, l'enregistrement permet de multiples expériences vocales. Il fait bien sûr apparaitre au fur et à mesure les difficultés de chacun. Il autorise aussi et surtout de nombreuses relations vocales. Par exemple, casque sur les oreilles, les participants s'écoutent lire le poème en l'enregistrant. Ils peuvent également (se) réécouter en cours de séance - et ainsi découvrir immédiatement une autre dimension de soi. Si cette écoute a lieu un certain temps après, le participant s'écoute au passé et, ce faisant, il réentend une écoute initiale du poème, qui était aussi un essai de compréhension autant qu'un premier essai de voix. L'enregistrement est aussi ce qui permet de faire un focus sur la matière du langage, notamment sur les éléments de diction - dont le souffle. Chez P. Fontana, enregistrer est ce geste qui ravive toujours plus de langage, qui fait même oublier le temps toujours compté du discours - et du cours de langue :

Mon travail, ce n'est pas de proposer un apprentissage phonétique. Les participants suivent par ailleurs des cours de FLE. D'une certaine façon, mon atelier est beaucoup plus pratique, plus concret. Je peux passer beaucoup de temps à faire sortir un son. Je peux m'arrêter, je peux passer l'atelier sur une seule phrase. Il y a une injonction joyeuse de ma part. Un rapport très pragmatique avec la langue : on arrive à faire quatre, cinq, six mots ensemble, avoir un souffle, le souffle de la ligne. (ibid.)

21 Chez P. Fontana comme chez d'autres concepteurs d'ateliers du dire en langue(s) étrangère(s), la lecture à voix haute comme essai de présence n'est pas simplement une incorporation minimale, une mise en voix, une expérience esthétique en langue étrangère, une lecture « expressive » (Rollinat-Levasseur, 2015, p. 232). Elle est la courroie de distribution entre faire atelier et faire langage, c'est-à-dire ce qui permet de pratiquer ensemble l'écoute continuée et l'essai de voix. Dans cette perspective, lire à voix haute n'est plus seulement une relecture attendue - récitation, mise en espace ou spectaclesynthèse. La lecture vocale constitue plutôt comme une unité de mesure dans l'atelier du dire - unité qu'il est possible d'additionner, de multiplier, d'ordonner selon des fonctions très variées. Comme chez M. Depeursinge, il faut noter de la part de P. Fontana une 
réserve concernant les oralisations ou les incorporations qui peuvent s'imposer par la force de la situation didactique. Le « vécu corporel dans la pratique d'une langue » (Louÿs \& Leeman, 2013) suppose une prudence méthodologique que les deux animateurs d'atelier semblent trouver dans un travail strictement vocal que le poème autorise : « Pour moi, ce qui est intéressant dans le travail de la voix, c'est que ce n'est pas intrusif. Contrairement à un atelier théâtre où, d'une certaine façon, je mets les gens en danger.» (Fontana \& Mouginot, 2017) Travailler avec la voix, les voix, présenterait ainsi un caractère moins engageant qui siérait mieux à certaines situations d'atelier. Cela ne signifie pas un moindre engagement du sujet du poème : lire à haute voix ce peut être déjà beaucoup de débordement de soi.

\section{Transformer la formation des enseignants en langue étrangère avec les arts du langage}

Les deux ateliers du dire évoqués ici nous semblent intéresser autant la didactique du FLE qu'interroger plus largement la formation initiale et continue des enseignants de français. Il y aurait beaucoup à gagner de "penser poème " non seulement pour concevoir des ateliers du dire qui durent au-delà du temps réglementaire mais aussi pour transformer la formation des enseignants au moyen d'essais de voix en langue étrangère favorisés par des parcours relationnels avec les œuvres de langage. Certains cursus de licence FLE contiennent dans leur curriculum l'expérience de découverte d'une langue étrangère - si possible éloignée de la langue maternelle de l'étudiant. Ce qu'il faudrait peut-être davantage viser sinon garantir, c'est l'expérience d'un véritable essai de subjectivation en langue étrangère, un essai transformé et non pas seulement une initiation. Dans cette perspective, il ne s'agirait pas seulement d'« élargir le répertoire méthodologique des enseignants de langue » (Beacco, 2007, p. 284) mais aussi et surtout de constituer un répertoire expérientiel fait de passages de voix, même très brefs.

Si les ateliers du dire questionnent à leur manière un langage qui «sert à vivre " (Benveniste, 1966, p. 217) comme enjeu d'enseignement et de formation, ils participent d'un signal émis déjà de longue date par la recherche. En linguistique, R. Jakobson (1963, p. 248) appelait à associer sur un plan théorique linguistes et littéraires. H. Meschonnic (2008, p. 47) a plusieurs fois réaffirmé la nécessité d'un enseignement généralisé d'une « théorie du langage » qui permette d'ouvrir tous les acteurs de l'éducation à une pensée du langage incluant une poétique des œuvres littéraires. Parmi les échos les plus récents, citons notamment avec A. Belhadj Hacen et I. Delcambre (2015, p. 18) le "défi de la professionnalisation des enseignants dans le domaine de la lecture-écriture, selon une perspective bi-plurilingue ». En didactique du FLE, G. Pierra (2006) plaide pour une formation théâtrale des enseignants avec des comédiens. Du côté du slam, le collectif $129 \mathrm{H}(2007$, p. 33) dresse le même constat: «Alors que l'intérêt de la mise en place d'ateliers d'écriture et d'expression slam est de plus en plus reconnu et promu [...], le nombre d'intervenants expérimentés à même d'animer des ateliers reste limité... ». Même s'il faudrait critiquer dans certains de ces discours des logiques contre-productives (spécialisation disciplinaire des arts du langage, séparation des temps d'apprentissage et de création, logique de l'intervenant extérieur), il faut cependant y lire l'expression d'un manque préjudiciable que les ateliers du dire en langue(s) étrangère(s) tentent de combler de manière souvent radicale : la réciprocité toujours à travailler entre langage et sujet(s). Dans notre conceptualisation, l'atelier du dire est l'un de ces lieux de langage où 
chaque sujet du discours travaille à son historicité - ce qui explique pourquoi aucun atelier ne ressemble à un autre. Au-delà des propositions et des situations d'atelier, il y a donc cette inquiétude d'une présence des sujets à l'écoute et à l'œuvre dans l'atelier, présence qui va jusqu'à travailler l'animateur lui-même :

je dois penser à une autre forme à l'atelier pour ne pas figer les choses, pour ne pas être absorbé par l'institution (le temps y contribue, je crois), continuer à produire du désir, à poursuivre entre les lignes, à trouver des chemins dérobés et à explorer la poésie de la langue, des langues. (Fontana, 2009-2011, 11/06/2010)

Quand P. Fontana se demande comment « continuer à produire du désir » au profit de son atelier de la voix, il est possible d'entendre comment susciter toujours plus d'« essayer dire » avec des poèmes qui sont faits de et pour ça. Cette question en cache d'ailleurs une autre : comment initier en atelier des essais de subjectivation langagière au moyen de poèmes, c'est-à-dire comment faire pour qu'un atelier du dire soit bien un atelier du sujet du poème? La question s'adresse également aux didactiques du français - qui s'en emparent progressivement. Certaines recherches en FLM, notamment en didactique de la poésie à l'école (Favriaud et al., 2009), s'intéressent avec profit à la spécificité de gestes professionnels - gestes didactiques et/ou conduites d'atelier - dont la force est d'ouvrir à de nouvelles situations relationnelles entre élèves, œuvres de langage et environnement d'apprentissage. Cependant, pour espérer un changement général et radical dans la vie des œuvres au cœur de ces didactiques, il reste non seulement à tirer des leçons d'expériences singulières souvent cachées ici ou là dans un bruyant maquis de pratiques, mais aussi et surtout à mettre en résonance - d'abord à travers la recherche - les nombreux ateliers du dire qui dessinent comme un atlas de la voix comme relation. C'est ce qu'à une échelle réduite nous avons entrepris dans notre thèse en didactique des langues et des cultures (2018), laquelle s'appuie sur l'analyse d'un corpus d'une demidouzaine d'ateliers en FLE. Dans un dialogue que nous avons voulu permanent avec la didactique du FLE, nous tentons de mettre au jour certaines spécificités de l'activité langagière proposée par ces expériences d'atelier. Cette activité que nous avons nommée « essayer dire » - en FLE - se signale par au moins trois marqueurs : une prise en compte très rigoureuse des historicités qui traversent tout être humain comme individu social et sujet de langage ; un continu expérientiel pleinement ancré dans un plurilinguisme posé comme "figure générale" (Boyer, 2010, p.13); un parcours relationnel avec une ou plusieurs œuvres de langage dont l'exemplarité en matière de modalités et d'intensités de subjectivation sert le projet transsubjectif de l'atelier. L'atelier du dire en langue(s) étrangère(s) comme atelier du langage crée et garantit du continu par les conditions mêmes de cet « essayer dire »- seule injonction programmatique qui lui est attachée. Ces conditions sont interdépendantes, se relancent sans cesse : il y a d'un côté le faire atelier qui relève des savoir-faire spécifiques de ceux qui conduisent l'atelier du dire (enseignants, animateurs, intervenants) et de l'autre le faire langage qui est l'engagement multiple - écoute(s), réénonciation(s), essais de voix - de ceux qui font l'expérience de l'atelier du dire (élèves, étudiants, apprenants, participants, écrivants, stagiaires et, dans une certaine mesure, les animateurs eux-mêmes). Par un détour qui est celui de toute odyssée, les ateliers du dire comme ateliers du «mode poème» viennent finalement rappeler que les œuvres de langage exemplaires sont celles qui font lire, écrire, dire autrement dit, qui font l'être humain à la fois plus critique et davantage partie prenante de la vie du langage. Plus important encore nous semble-t-il, les ateliers du dire font découvrir ou reconnaitre combien ce que le poème fait, rien d'autre ne peut le faire à sa place - rien ni personne. Dans cette optique, peut-être les ateliers du dire sont-ils moins 
l'occasion d'un « essayer dire " que d'un essayer vivre autrement, spécifique à l'« acte de littérature » évoqué par H. Meschonnic (2008, p. 69). La tacite reconduction de nombre d'expériences d'ateliers du dire sur des périodes relativement longues révèle que ces pratiques sont parvenues à prendre une place singulière à côté d'interactions ou d'environnements didactiques plus conventionnels. Il faut souhaiter la même longévité aux recherches qui accompagneront ces pratiques, voire les amplifieront.

\section{BIBLIOGRAPHIE}

ADEN, J. (2013). « De la langue en mouvement à la parole vivante : théâtre et didactique des langues ». Langages 192, p. 101-110. En ligne : https://www.cairn.info/revue-langages-2013-4page-101.htm.

BAUDELAIRE, C. (2003) [1869]. « L'étranger ». In : Baudelaire, C., Le Spleen de Paris. Petits poèmes en prose. Paris : Librairie générale française.

BEACCO, J.-C. (2007). L'approche par compétences dans l'enseignement des langues. Enseigner à partir du CECRL. Paris : Didier.

BECKETT, S. (1991) [1982]. Cap au pire. Trad. par É. Fournier. Paris : Minuit.

BELHADJ HACEN, A. \& DELCAMBRE, I. (dirs) (2015). Littéracies et plurilinguismes. Quelles pratiques? Quels liens? Paris : L'Harmattan.

BENVENISTE, É. (1966). « De la subjectivité dans le langage ». In : Benveniste, É., Problèmes de linguistique générale I. Paris : Gallimard, p. 259-260.

BERNARDET, A. (2012). «L'inconnaissance du littéraire. L'ethos critique comme pédagogie ». Polartnet. En ligne : http://polartnet.free.fr/textes/textes_polart/inconnaissance.pdf.

BOYER, H. (2010). « Diversité et pluralité linguistiques : les chantiers de la sociolinguistique ». Culture et recherche 124, p. 13-14.

CADRE EUROPÉEN COMMUN DE RÉFÉRENCE POUR LES LANGUES. (2001). Apprendre, enseigner, évaluer. Paris : Conseil de l'Europe/Didier.

CERTEAU, M. de (1990) [1980]. L'invention du quotidien. 1. Arts de faire. Paris : Union générale d'édition.

COLLECTIF 129H (2007). Petit guide méthodologique pour l'animation d'ateliers slam. Paris : 129H productions.

DEPEURSINGE, M. (2018). L'éducation poétique par l'illisible. Enquête sur les conditions de la lecture en démocratie. Thèse en lettres. Université de Lausanne.

DEPEURSINGE, M. \& MOUGINOT, O. (2017). « Un atelier de "l'illisible" : entretien avec M. Depeursinge ». Atelit, carnet de thèse numérique. En ligne : https://atelit.hypotheses.org/1552. DESSONS, G. (2015). La voix juste. Essai sur le bref. Paris : Manucius. 
DOS SANTos, G. (2013) « "Je est un autre”. L'expérience du bilinguisme et du théâtre pour enseigner et apprendre une langue ». Langages 192, p. 111-117. En ligne : https://www.cairn.info/ revue-langages-2013-4-page-111.htm.

FAVRIAUD, M. et al. (2009). « Nourrissage, amorçage et gestes professionnels dans la production poétique au cycle 2 de l'école primaire ». Repères 40, p. 175-199. En ligne : https:// journals.openedition.org/reperes/340.

FONTANA, P. (2009-2011). Cahiers d'Emmaüs. En ligne : http://cahiers-emmaus.blogspot.com. fONTANA, P. (2013) Luca Babel. Parcours dans l'œuvre de Ghérasim Luca [CD]. Production : Khiasma, Patrick Fontana, Association Emmaüs Solidarité.

FontANA, P. \& MOUGinot, O. (2017). « Ateliers "lectures de bouches" : entretien avec P. Fontana ». Atelit, carnet de thèse numérique. En ligne : https://atelit.hypotheses.org/1582.

GODARD, A. (dir.) (2015). La littérature dans l'enseignement du FLE. Paris : Didier.

GODARD, A., HAVARD, A.-M. \& ROLLINAT-LEVASSEUR, È.-M. (dirs) (2011). L'expérience de lecture et ses médiations. Réflexions pour une didactique. Paris : Riveneuve.

JAKOBSON, R. (1963). Essais de linguistique générale. Trad. de l'anglais par N. Ruwet. Paris : Éd. de Minuit.

JOUBERT, C. (2015). Critiques de l'anglais. Poétique et politique d'une langue mondialisée. Limoges : Lambert-Lucas.

LOUŸS, G. \& LEEMAN, D. (coords) (2013). « Le vécu corporel dans la pratique d'une langue ». Langages 192. En ligne : https://www.cairn.info/revue-langages-2013-4.htm.

LUCA, G. (2001) [1985]. Héros-Limite suivi de Le Chant de la carpe et de Paralipomènes. Paris : Gallimard.

MARTIN, S. (2005). «Faire œuvre avec les œuvres ». Le français aujourd'hui 149, p. 67-73. En ligne : https://www.cairn.info/revue-le-francais-aujourd-hui-2005-2-page-67.htm.

MARTIN, S. (2016) «Je n'enseigne point, je raconte. La voix, la relation : réflexions théoriques et didactiques ». Carnets 8. En ligne : http://carnets.revues.org/1926.

MATHIS, M. (2013). Identités plurilingues et création textuelle en français langue étrangère : une approche sociolinguistique d'ateliers d'écriture plurielle. Thèse en sciences du langage : Université d'Avignon et des Pays du Vaucluse/Simon Fraser University.

MESCHONNIC, H. (2006) [1989]. La rime et la vie. Paris :Gallimard.

MESCHONNIC, H. (2008). Dans le bois de la langue. Paris : L. Teper.

MESCHONNIC, H. (2009) [1982]. Critique du rythme. Anthropologie historique du langage. Lagrasse :

Verdier.

MOUGINOT, O. (2018). Les ateliers du dire (lectures, écritures, littératures): enjeux et expériences de la voix en langue(s) étrangère(s). Thèse en didactique du français et des langues : Université Sorbonne Nouvelle Paris 3.

NOIRET, G. (2011). Autoportrait au soleil couchant. Bussy-le-Repos : Obsidiane.

PAÏNI, Ph. (2005). «Entendre clairement ce qui nous trouble ». Le français aujourd'hui 150, p. 45-53. En ligne : https://www.cairn.info/revue-le-francais-aujourd-hui-2005-3-page-45.htm.

PIERRA, G. (2006). Le corps, la voix, le texte. Arts du langage en langue étrangère. Paris : L'Harmattan. 
PRIGENT, C. (2012). « (du neuf sur la schize)». In : Prigent, C., La Vie moderne. Un journal. Paris :

P.O.L., p. 43

ROLLINAT-LEVASSEUR, È.-M. (2015). « La littérature en acte : voir, entendre, ressentir ». In : Godard, A. (dir.), La littérature dans l'enseignement du FLE. Paris : Didier, p. 222-264.

SIOUFFI, G. (2007). «Du sentiment de la langue aux arts du langage ». Études de linguistique appliquée 147, p. 265-276. En ligne : https://www.cairn.info/revue-ela-2007-3-page-265.html.

Velter, A. (2001). « Parler apatride. Préface d'André Velter ». In : Luca, G., Héros-Limite suivi de Le Chant de la carpe et de Paralipomènes. Paris : Gallimard, p. I-XVI.

VORGER, C. (2011). Poétique du slam. Néologie, néostyles et créativité lexicale : de la scène à l'école. Thèse en sciences du langage : Université de Grenoble.

\section{RÉSUMÉS}

Qu'il s'agisse d'ateliers d'écriture, de slam ou de théâtre conduits en situation d'acquisition ou d'apprentissage $d u$ français comme langue étrangère, ces expériences langagières ont pour dénominateur commun d'être des invitations à "essayer dire" - expression de S. Beckett. L'analyse de ces ateliers du dire en langue étrangère révèle que ce sont souvent des ateliers avec la littérature mais aussi et surtout des ateliers du poème. Cette fréquence n'est pas fortuite : chaque poème joue un rôle précis dans la mise en œuvre de l'atelier du dire défini comme parcours expérientiel et essai de subjectivation en langue étrangère. À ce titre, de telles pratiques plurilingues nous semblent devoir intéresser la didactique du FLE. Nous illustrerons notre proposition en rendant compte de deux ateliers du poème. Conduit en Suisse par un enseignant de français, le premier est dénommé « atelier de l'illisible » et propose de modifier les conditions d'écoute d'un poème difficile en faisant se questionner des adolescents étrangers sur la signification de leur propre activité de compréhension. Atelier de lecture de poèmes à voix haute, les «lectures de bouches " s'adressent à des adultes nouvellement arrivés en France. Par des pratiques de lecture chorale et un usage particulier de procédés d'enregistrement numérique, cet atelier animé par un comédien vise à susciter des passages de voix d'une langue à l'autre, d'un participant à l'autre, ou encore d'un poème à un autre.

Whether it is writing, slam or theatre workshops, conducted in situations of acquisition or learning French as a foreign language, these language experiences have as a common denominator to be invitations to "try say" - to quote S. Beckett. The analysis of such workshops in foreign languages reveals that many are not only workshops with literature but also and especially workshops of the poem. This frequency is not fortuitous: each poem plays a precise role in the implementation of the workshop defined as an experience course and a try of subjectivation in a foreign language. Such multilingual practices should attract the attention of researches in teaching and learning French. We will illustrate our proposal by reporting on two workshops of the poem. The first one, which took place in Switzerland, is called "workshop about illegible poems". A teacher proposes to change the conditions of listening to a difficult poem by making foreign adolescents ask themselves questions about the meaning of their own understanding activity. As a poem-reading workshop, the "mouths that read" are dedicated to newly arrived adults in France. Through choral reading practices and a particular use of digital recording methods, this workshop led by an actor aims to make voice(s) pass from one language to another, from one participant to another, or from a poem to another. 
INDEX

Mots-clés : didactique des langues, français langue étrangère (FLE), arts du langage, littérature, poésie, poème, lecture, atelier

Keywords : teaching and learning foreign languages, French as a foreign language, language arts, literature, poetry, poem, reading, workshop

\section{AUTEUR}

\section{OLIVIER MOUGINOT}

Université Sorbonne-Nouvelle - Paris 3, DILTEC, EA 2288, F-75005, France 